\title{
BECKETT ET LES MOTS DE L'AFFECT
}

\begin{abstract}
A b stract. Lis Jerzy, Beckett et les mots de l'affect [Beckett and the affective words]. Studia Romanica Posnaniensia, Adam Mickiewicz University Press, Poznań, vol. XXIX: 2003, pp. 21-29, ISBN 83-232-1232-5, ISSN 0137-2475.
\end{abstract}

The subject of this article is analysis of Beckett's affective language in his play Comment c'est. The author shows similarity of many of the playwrights procedures to Ludwig Wittgenstein's philosophical conceptions.

La réflexion sur l'écriture confrontée avec l'affect en vue d'envisager une certaine humanité de Beckett permet d'ouvrir, me semble-t-il, de nouvelles perspectives pour la lecture de cette oeuvre difficile et énigmatique. En effet, elle ne cesse de dérouter et de surprendre aussi bien les lecteurs que les spectateurs, et, certainement, elle est toujours à redécouvrir. La relation écriture-affect situe le débat au niveau d'une autre relation, plus importante celle-ci, à savoir langage-psychologie, concepts ô combien beckettiens, dont le sens généralisateur renvoie aux propositions philosophiques de Ludwig Wittgenstein formulées en 1922 dans Tractatus logico-philosophicus et reformulées à l'infini, voire modifiées dans ses écrits postérieurs dont, entre-autres, Philosophical Investigations, paru à titre posthume en 1953.

Il n'est pas de mon propos de démontrer les différences entre le «premier» et le «second» Wittgenstien qu'illustrent bien les textes mentionnés, mais de voir comment ses investigations, allant de la philosphie du langage à la philosophie de la psychologie, sont proches de celles de Samuel Beckett. Sur le ton un peu métaphorique on pourrait parler des ressemblances de famille. En signalant ci-dessus les deux niveaux de relations, et sans que cela soit voulu, je fais appel non seulement à la théorie des prototypes, mais aussi aux tendances classificatrices propres aux philosophes et aux psychologues. En tant qu'objets des classifications, le langage et l'affect tendent vers l'expression d'un état élémentaire censé décrire l'essence des choses. Wittgenstein était conscient que le langage a ses limites et 
n'exprime qu'en partie ce qu'on voudrait dire. Sa conception de jeux de langage ${ }^{1}$ et, en général, sa théorie de l'image relèvent du caractère multiforme du langage qui doit répondre non seulement à la question de quoi est fait un objet, mais également de quoi est fait son image, et par conséquent ce à quoi on pense en parlant.

Supposer qu'une image désigne l'usage unique ou globalisant du mot est illusoire. L'image qui accompagne un mot ou une expression n'est qu'un usage possible, ce qui nous permet de constater qu'il s'agit d'un objet à sens multiple ${ }^{2}$. Le cas extrême serait l'image nue qui n'est applicable à rien, donc celle qui n'a aucun sens. La septième et dernière proposition de Tractatus: «Ce dont on ne peut parler, il faut le taire» $(T, 7)$, résume le mieux comment, tout en étant notre outil quotidien, le langage reste imparfait et incompréhensible. Cela est particulièrement visible dans la construction même de Tractatus dont les six premières thèses principales autour desquelles gravitent les propositions complémentaires, forment un système homogène où la place centrale est attribuée à la question des relations entre la pensée et le monde, et au rôle que joue dans ces relations le langage ${ }^{3}$. Sans trop simplifier les choses il faut remarquer seulement que, comme le dit Wittgenstein, les limites de notre langage constituent les limites de notre univers (cf. $T, 5.6)$. Or, les limites du langage sont déterminés par les conditions logiques de toute symbolisation ce qui correspond chez Wittgenstein à la notion kantienne des «intuitions transcendentales aprioriques».

L'une des notions introduites dans Tractatus, et qui nous intéresse dans le débat sur l'affect, est celle du sujet dont on dit que le monde est «son» monde (cf. $T, 5.641$ ). Le sujet wittgensteinien est un sujet métaphysique, donc ce n'est pas une personne concrète, vous ou moi, mais l'ensemble de contenu mental ou plutôt celui d'idées et de phrases intelligibles, où les consciences individuelles ne font que participer. Et puisque la structure élémentaire de la pensée et celle de la réalité sont identiques, Wittgenstein a proposé en effet une théorie phénoménologique du langage. La manière non restrictive de l'emploi du terme «phénomène» permet au philosophe de parler de toutes sortes de phénomènes psychologiques et de conclure que les états d'esprit ainsi que les passions sont perceptibles sur le visage de l'individu. Cependant certains segments de notre langage, notamment la terminologie psychologique, nous empêchent de décrire la réalité, car il est pratiquement impossible d'atteindre l'esprit de l'autre et de savoir exactement quel est son état. Le mot a le sens qu'on lui a attribué d'où il s'ensuit qu'on ne peut pas tout exprimer. Et inversément, l'usage constamment exercé du mot permet de lui donner une signification attendue.

${ }^{1}$ Wittgenstein a très bien décrit les jeux de langage dans son Cahier bleu (1933-1934); cf. L. W it t g e n s t e i n, The Blue and Brown Books, éd. Basil Blackwell, Oxford 1958.

${ }^{2}$ Cf. J. W i e r t l e w s k a, Teoria obrazu w „Dociekaniach filozoficznych", dans: Maciej Soin (dir.), Wittgenstein w Polsce, éd. Wydawnictwo IFiS PAN, Warszawa 1998, pp. 123-134.

${ }^{3}$ Cf. B. W o l n i e w i c z, Fakty i rzeczy. Wstęp do pierwszej filozofii Wittgensteina, éd. PWN, Warszawa 1968, p. 28. 
L'aspect anthropologique de la «seconde» philosophie de Wittgenstein témoigne en effet de l'intérêt du philosophe pour la psychologie et cela malgré le chaos notionnel qui lui est propre. Le langage est devenu l'un des éléments de l'existence et de l'activité humaines et a fini par absorber diverses formes d'expression, y compris métaphores et comparaisons, ce qui nous oblige à juger sur les phénomènes à partir d'images fixées par lui ${ }^{4}$. Le psychologue réfléchissant sur l'affect élémentaire est amené à décrire les émotions à l'aide des notions que nous considérons comme convenables pour leur description. La classification des émotions est donc le fait du langage surtout lorsque nous voulons expliquer les relations de subordination - l'indignation est-elle une variété de colère ou seulement son aspect? En dressant des listes des affects, les psychologues recourent presque toujours à la figure rhétorique de synecdoque, pratique, semble-t-il, pour la description soit d'une classe d'émotions, soit d'une émotion particulière 5 .

Si l'on accepte généralement qu'on devine les émotions grâce aux signaux affectifs (sourire, pleur, etc.), on voudrait savoir quels sont les signaux linguistiques qui informent les autres sur le type de l'émotion éprouvée. Il est évident que conformément aux principes sociaux de la communication, tout langage s'est constitué son propre système de conditions pour la verbalisation et le codage des émotions. Leurs noms sont une représentation réflexive de l'affect et, comme toute autre appellation, ils sont l'effet d'une décision arbitraire. Chaque culture dispose d'un important arsenal linguistique qui permet d'exprimer les expériences en termes non émotionnels et de manière métaphorique. Les théories modernes des émotions deviennent de plus en plus tributaires de l'interprétation, voie par laquelle l'expérience vécue est mise en ordre. L'un des théoriciens de l'affect, Richard A.Shweder, trouve que les émotions ne sont ni objets, ni notions, ni non plus termes de langage, mais des structures narratives qui donnent la forme et le sens aux sensations somatiques et affectives ${ }^{6}$. Les interprétations abstraites ne sont guère des événements qui précèdent l'émotion, mais ils constituent une partie inséparable de chaque structure narrative. Dans chaque récit (qu'il soit intitulé «peun» ou «honte») l'expérience affective se présente comme la perception concernant le «moi». Ainsi pour pouvoir constater que l'événement somatique et affectif a eu lieu, il faut le percevoir et, par conséquent, répondre par une réaction.

${ }^{4}$ Cf. P. M.S. H a c k e r, Insight and Illusion. Themes in the Philosophy of Ludwig Wittgenstein, éd. Clarendon Press, Oxford 1986. La rêférence renvoie au texte paru dans la traduction polonaise: A. Chmi e lewski, A. Orze ch ow s k i (réd.), Metafizyka jako cień gramatyki. Późna filozofia Ludwiga Wittgensteina. Wybór tekstów, traduction collective, éd. Wydawnictwo Uniwersytetu Wrocławskiego, Wrocław 1996, pp. 40.

${ }^{5}$ Cf. P. Ek m a n, R. J. D a vi d s o n (réd.), The nature of emotion. Fundamental Questions, éd. Oxford University Press Inc., Oxford 1994. Je me rêfêre à l'édition polonaise de cet ouvrage dans la traduction de Bogdan Wojciszke: Natura emocji. Podstawowe zagadnienia, éd. Gdańskie Wydawnictwo Psychologiczne, Gdańsk 1998, p. 17.

${ }^{6}$ Ibidem, pp. 36-47. 
Il est intéressant de constater que cette théorie rejoint celle que Wittgenstein a appelée «jeux de langage» ou «forme de la vie» («form of life») ${ }^{7}$ dont l'essence consiste à décrire le monde. J'y reviendrai encore plus loin. On ne peut pas sous-estimer le caractère très suggestif de la mystique wittgensteinienne qui, tout en valorisant quelque qualité inexprimable du monde, s'intéresse à la chose la plus simple, à savoir: le goût de vivre. La réponse à la question élémentaire comment c'est quand on vit peut être donnée par quelqu'un qui a essayé de goûter à l'existence. Regardons donc du côté de Beckett pour savoir comment c'est dans Comment c'est.

En analysant l'attitude de Beckett vis-à-vis du langage affectif, il faut être conscient qu'à l'étape de Comment c'est, l'écrivain est beaucoup plus constructif en ce qui concerne le rapport entre la forme et le contenu. La forme n'imite plus l'existence, mais elle l'adapte ou l'apprivoise même malgré la dissonance entre l'ordre voulu de la première et le chaos de la seconde'. C'est d'autant plus intéressant que les préoccupations du romancier semblent avoir peu changé depuis la publication de la trilogie. Les questionnements sur l'existence dans la perspective de la mort, voire les débats métaphysiques, les réflexions à propos de l'intellect, tout est là pour mettre en relief la lutte de l'homme contre le temps et l'espace, et surtout contre le langage dont il ne peut se libérer et qui est à la fois son unique espoir. C'est donc par l'intermédiaire du langage que Beckett espère dompter le désordre mental de l'homme. Le sujet lyrique exploite toutes les possibilités de langage pour évoquer sa situation par rapport à l'univers qui l'entoure. Bien que, comme ce fut le cas chez Wittgenstein, on ne puisse sortir avec le langage en dehors de lui, le lecteur est constamment forcé à interpréter, expliquer, choisir, sans jamais comprendre le sens véritable des mots. La tactique beckettienne consiste à structurer le récit selon les modalités de globalisation du discours. Puisque le langage n'exprime qu'en partie notre situation, le romancier recourt à différents registres, accumule des propos et hypothèses qui donnent l'illusion d'exprimer la réalité alors qu'ils témoignent de notre échec. Le scepticisme de Beckett est du même ordre que celui de Wittgenstein - afin que le problème soit résolu, il doit cesser de nous préoccuper, alors que dans Comment c'est le lecteur est constamment bombardé par le retour obsessionnel des problèmes insolubles ou sans réponse, car ils échappent aux fonctions référentielles du langage.

Le titre du texte beckettien est à la fois une constatation et une question sur les formes de la vie et suggère de manière indirecte qu'imaginer le langage c'est le lier avec quelque activité. Comment c'est s'offre comme une entreprise de décrire

${ }^{7}$ Il s'agit de l'ensemble des dispositions pour de différents comportements, liés réciproquement - mines, gestes, actions; cf. A. Chmie lewski, A. Orzechowski, Metafizyka jako cień gramatyki, op. cit., p. 92.

${ }^{8}$ Toutes les citations qui vont suivre renvoient à l'èdition suivante: S. B e c ke t t, Comment c'est, éd. Les Éditions de Minuit, Paris 1961.

${ }^{9}$ Cf. M. K ę d z i e r s k i, Samuel Beckett, éd. Wiedza Powszechna, Warszawa 1990, p. 82. 
l'existence, de la rédiger dans le but de voir en quoi consiste «être et ne pas être passé présent futur et conditionnel» (p. 59). Inutile d'y chercher la terminologie précise de l'affect, quelques emplois de «bonheur», «malheur», "amour» informent peu sur l'état affectif du personnage. Au lieu de nommer, Beckett avance plutôt des thèmes relationnels des affects élémentaires qui signalent que telle ou autre émotion a été déclenchée. En effet, c'est au lecteur qu'appartient le devoir de déduire à partir de différents vocables, groupes de mots ou encore métaphores quelle émotion est en jeu. Bien des mots sont dès le départ empreints d'affectivité, mais ils ne décrivent pas la situation du personnage, parce qu'il est difficile de déterminer ce qui, dans la relation entre le sujet et l'objet de l'émotion, est indispensable pour que l'expérience affective ait lieu. L'homme a besoin d'images qui associent des mots puisés dans différents registres de langage et qui expriment de simples vérités (martyriser c'est autre chose qu'être martyrisé).

Force est de convenir que le moindre incident et la moindre mention d'un objet peuvent devenir pour Beckett le point de départ d'une réflexion sur la nature des relations affectives. Cependant il est à noter que l'auteur n'attribue presque jamais de valeur fixe aux mots qui décrivent l'affectivité, car tout ce qui est figé, sonne faux. Cela est d'ailleurs conforme avec la perception par l'individu des situations qui s'étendent, chez Beckett, entre deux extrémités que sont le bien et le mal. $\mathrm{La}$ diversité des états et incidents psychiques que ses mots décrivent, résulte du besoin d'échapper à la fixité. Ainsi remarque-t-on souvent la facilité avec laquelle l'auteur passe des émotions aux sentiments, sensations ou humeurs en leur attribuant les qualités émotionnelles qu'ils n'ont pas. Le sentiment de la faim, la fatigue sont les réactions du corps dans la même mesure que la sensation de l'incertitude ou la surprise sont les réactions de l'esprit.

Beckett joue librement des antinomies, juxtapose les sens contraires des mots pour révéler ce qui peut être à la fois agréable et déprimant dans le cadre du même affect. Le fameux bonheur malheur beckettien est tout au long du récit expliqué comme la somme des émotions positives et négatives: la douleur physique n'exclut pas la joie («je hurlais au secours avec une fois sur cent un certain bonheur», p. 58), chanter pleurer (p. 106) en tant que symptômes contradictoires de la peur et de la joie, peuvent désigner la même expérience émotionnelle. Il n'y a rien de plus pénible que d'éprouver quelque chose et de ne pas savoir le nommer. Plus d'une fois le parleur se voit obligé de recourir au discours de remplacement, de contourner l'essentiel pour se prouver qu'il sait nommer: «le je dis comme je l'entends» (p.ex. pp. 20, 21, 28).

L'une des particularités de Beckett est d'être revenu dans Comment c'est aux choses élémentaires, voire à ce qui peut être nommé, même au risque d'être ambigu. Le romancier simplifie des notions et des mots et restreint leur signification au niveau du paradigme. Il évite ainsi l'excès affectif, car son langage construit alors un discours non moins hypothétique qu'analogique. Le positif et le négatif, la voix et le silence, le bonheur et le malheur, le bourreau et la victime, finissent par 
désigner l'intentionnalité émotionnelle identique ${ }^{10}$. Des mots-clés comme boue, sac à charbon, boîtes, ouvre-boîte, se réferrent non seulement à la souffrance du parleur ou à son inconfort physique ou psychique, mais aussi à son bien-être, parce que c'est à lui que les objets mentionnés appartiennent. Peu importe au fond si l'emotion est positive ou négative, si une olive se confond avec une cérise (cf. p. 25), si le sac est rempli de boîtes ou qu'il est vide, - le personnage ne signale aucune préférence pour l'un ou l'autre. L'essentiel est de savoir les mots et nommer non pour comprendre l'univers, mais pour saisir les lois qui régissent sa description.

Or, imaginer le langage veut dire, pour Beckett comme pour Wittgenstein, imaginer une forme de la vie, ce qui suggère ni plus ni moins que le langage est étroitement lié avec quelque modèle d'activité ${ }^{11}$. En tant qu'ensemble des dispositions de l'individu, la forme de la vie systématise certains comportements humains enchaînés dans une série logique qui se résume ainsi: activité (expérience), réaction mimique et gestuelle, réaction mentale et interprétation (désignation). Ce jeu de langage correspond dans les grandes lignes à l'organisation fonctionnelle des émotions. Au niveau intrapersonnel, toute réaction émotionnelle comprend trois symptômes: l'expérience de l'émotion (choc, sensation physique, souvenir), réaction mimique et vocale, réaction physiologique. Au niveau interpersonnel ils sont complétés par l'expression qui détermine le caractère des transactions entre deux individus ${ }^{12}$. Le souvenir d'une scène avec Pim pourrait servir ici d'un bon exemple: l'instant solennel (première tranche) est suivi de l'étape fertile en vicissitudes et péripéties (seconde tranche), laquelle aboutit à ... entre autres extravagances dont l'usage de la parole (pp. 88-95).

L'usage de la parole n'est ici que symbolique, car l'extravagance mentionnée concerne aussi la parole mécanique (pan vlan, p. 101) accordée gracieusement à l'interlocuteur (je ne vais pas me fatiguer à lui demander..., p. 101). En revenant aux thèmes relationnels, donc à une description par le langage de ce qu'on entend par telle ou autre émotion éveillée par l'expérience avec l'autre, Beckett s'épuise à thêâtraliser les affects tel que colère, amour, espoir, tristesse, bonheur, compassion, honte, afin de pouvoir les nommer. Il est intéressant que l'auteur procède par séries associatives dont Wittgenstein fut un grand amateur: à une main ajouter une autre main, puis doigts, ongles monstres, boue, cheveux, «quelques vieux mots par-ci par-là les ajouter les uns aux autres faires des phrases» (p. 164). Faute de mots, il est impossible de décrire l'expérience, d'en retenir une image qui soit conforme aux principes de formes de la vie. Puisque chez Beckett ce sont les émotions qui font appel aux mots, il est évident qu'il n'y a rien de plus insupportable que d'éprouver quelque chose et ne pouvoir l'appeler. Visiblement, ne souffre qui possède la parole.

${ }^{10}$ Cf. Natura emocji. Podstawowe zagadnienia, op. cit., pp. 58-60.

$"$ Cf. H. Le Roy Finch, Forms of Life [Formy życia], dans: A. Chmielewski, A. O r z e c how s k i, Metafizyka jako cień gramatyki, op. cit., p. 97.

${ }^{12}$ Cf. Natura emocji. Podstawowe zagadnienia, op. cit., p. 112. 
En soutenant une thèse que chez les personnages beckettiens l'existence ne vaut que si elle est garante de la fluidité du passage interminable émotion - parole émotion, la suppression d'un élément de cette relation rend stérile toute communication avec l'autre. Ainsi le rapport entre Pim et le parleur est envisagé comme une suite déchaînée d'actes de cruauté et de cris dans le but de personnaliser le langage ${ }^{13}$ avant que d'autres émotions et d'autres paroles soient mobilisées: «le nommer le dresser le couvrir jusqu'au sang de majuscules romaines me gaver de ses fables nous unir pour la vie» (p. 97). Au fond la relation avec l'autre consiste à éveiller réciproquement des passions et des désirs. Une fois la première expérience vécue, l'image du quidam quelconque (donc d'un individu qu'on ne peut pas désigner avec précision) change radicalement du point de vue affectif - «il est mieux mieux qu'hier moins laid moins bête moins méchant moins sale moins vieux moins malheureux...» (p. 12).

Beckett est entièrement conscient qu'en dehors de règles de grammaire (selon la conception wittgensteinienne) l'emploi conventionnel du langage n'exprime pas grand'chose. Il se comporte donc comme un métaphysicien qui rejette nos conventions de l'usage des mots et recommande une forme de la description ${ }^{14}$. Cette description prend dans le texte analysé le caractère d'un examen douloureux de ce qui est compréhensible et ce qui ne l'est pas, mais doit être pour que la réponse à la question «comment c'est» soit envisageable. Que se passe-t-il donc quand les mots font défaut?

Nous savons qu'au niveau intrapersonnel les traces de l'expérience émotionnelle sont signalées par les réactions mimiques, gestuelles et physiologiques, suivies de pensées, lesquelles sont notre manière individuelle de rationaliser l'affect. Or, Beckett est très sensible à la dernière proposition de Tractatus logico-philosophicus et compense le manque de mots par la «parole mécanique» ou d'autres formes d'extériorisation de l'état vécu. Lorsque le parleur déclare: «je ne sens pas ma fatigue elle s'exprime néanmoins» (p. 64), il est en train de verbaliser l'affect qui échappe pourtant à la verbalisation. L'émotion est nommée, car elle est perceptible par l'autre. Nous revenons ici à la description par le langage où le personnage s'attaque aux mots et à leur grammaire, et crée ainsi de nouveaux sens et faits. Il ne fait pas de doute que l'entreprise s'avère comme une nécessité: «trouver des mots encore alors qu'ils sont tous dépensés brefs mouvements encore du bas du visage [...] aucun son c'est mes mots qui font ça ça fait mes mots je m'endormirai encore dans l'humanité» (p. 69).

${ }^{13}$ Tout mutisme imposé ou la perte volontaire du langage relèverait de la reconnaissance de son caractère non personnel; cf. G. D u rozo i, Beckett, coll. Prêsence littéraire, éd. Bordas, Paris Montrêal, p. 175 .

${ }^{14}$ Cf. A. Ch mi e lew ski, A. Orze chow s ki, Metafizyka jako cień gramatyki, op. cit., p. 74 . 
«Le peu que ie dis aucun son le peu que ie vois» (p. 32) ${ }^{15}$ serait non seulement l'application des règles wittgensteiniennes en vue de maîtriser le langage, mais le moyen de comprendre le monde et de ressentir comment une chose nommée pourrait l'expliquer. Rien ne décrit mieux le drame du parleur que cette tentative désespérée de lutter contre le mutisme et l'oubli, ce besoin de répéter et de composer des mots en phrases, de les murmuer et associer à l'infini selon un ordre logique. Et si ce n'est pas suffisant, recourir aux gestes, toucher, se débattre pour voir comment c'est. Certes, Beckett excelle dans l'expression théâtrale de la gestuelle - porter la main vers le visage, l'agiter, se la poser sur le visage, main qui serre le sac, main qui s'ouvre et se referme dans la boue - autant de gestes qui sont des mots muets et qui remplacent le langage. Ne dit-il pas que: «j'en ai besoin d'un mot de ma main» (p. 27)?

Si l'on considère la gestuelle imaginée dans Comment c'est comme un mécanisme adaptatif de l'interaction avec l'entourage, Beckett a certainement trouvé un moyen extraordinaire pour la description des émotions ${ }^{16}$. Il joue incessament avec des gestes qui, tout en reflétant la richesse, voire la complexité émotionnelle du personnage, sont dessinés comme une forme de substitution du langage. L'écrivain ne fait plus appel aux concepts figés des émotions (par exemple il ne dit jamais: Pim est la tristesse même ou Bom incarne le bonheur, etc.), mais puise dans les thèmes relationnels, c'est-à-dire dans l'idée que nous nous faisons de la représentation du comportement affectif. Beckett met en scène les composants élémentaires des émotions dont le sens varie en fonction de la situation qui est en jeu. C'est dire qu'avant que l'affect soit visualisé, le personnage beckettien est toujours en train d'évaluer sa position vis-à-vis de l'autre, de cet autre qui est la source des réactions positives ou négatives ${ }^{17}$. A l'instar des mots dans un discours, les gestes sont également soumis à la loi associative de composition et s'appuient sur une simple relation mathématique (A à $\mathrm{B} B$ à $\mathrm{C}$ ).

L'échangeabilité de gestes constitue l'essence de la description de l'affect chez Beckett. Ses personnages sont suspendus entre deux situations émotionnelles extrêmes («chacun d'entre nous ne connaît personnellement que son bourreau et sa victime», p. 185) ou bien, chacun à leur manière et à l'aide des gestes contradictoires, ils essaient de se retrouver par rapport à autrui. C'est pour cela, en suivant souvent le bon sens de la grammaire witgensteinienne, que Beckett recourt aux

${ }^{15}$ C'est nous qui soulignons.

${ }^{16}$ En psychologie l'émotion est un mécanisme adaptatif de l'interaction avec l'entourage et fonctionne comme un fond des états à caractère existentiel; cf. Natura emocji. Podstawowe zagadnienia, op. cit., pp. 75-80.

${ }^{17}$ Les psychologues trouvent que c'est le sentiment de l'incertitude qui stimule cet acte de reconnaissance. L'individu devient alors conscient de ce qui l'attend de la part de l'autre; cf. Natura emocji. Podstawowe zagadnienia, op. cit., p. 179; cf. aussi B. Parki n s o n, A. M. Colman, Emotion and Motivation, éd. Routledge, 1994, traduit en polonais par Izabela Sowa sous le titre: Emocje i motywacja. éd. Zysk i S-ka, Poznań 1999, p. 62 et suivantes. 
modèles de l'usage des mots et leur attribue le sens conforme tant à la situation qu'il est en train de décrire, qu'à ce qui se présente comme l'envers et l'endroit des choses (on répéterait volontiers la formule obsessionnelle du parleur - «c'est selon ce qu'on entend»). La construction «on boit on donne à boire» (p.189) illustre les mêmes règles de langage et décrit deux situations, voire deux gestes analogues, alors que leur intentionnalité affective est différente. Il en est de même pour beaucoup d'autres constructions qui connotent la variété de la gestuelle becketienne - «souffrance cruauté» (p.189), "amour peur», "chanter crier» (p.102), «martyriser être martyrisé». Des expressions banales telles que «tenir la tête» et «tenir le sac», rapprochées dans le texte les unes des autres, constituent le véritable jeu de l'affect qui échappe au langage au sens habituel du terme.

Les mots et les gestes sont souvent ramenés à l'état d'abstraction, mais Beckett leur impose un ordre logique, les associe en couples qui mettent en relief l'ambivalence affective de l'homme comme si l'affectivité même se mesurait par l'intensité des expériences passées et présentes. Le rythme du texte reflète le rythme de la vie comme dans ce passage: «un peu d'affection trouver quelqu'un que quelqu'un vous trouve enfin vivre ensemble collés $[\ldots]$ s'aimer un peu aimer un peu sans être aimé» (p.117). On a très souvent l'impression que Beckett fait noyer son lecteur dans un océan de mots et de gestes dont le rôle consiste à nous épargner la situation extrême où il n'y aurait que «bribes de vie», «loques de vie». L'incompatibilité ou l'inconvenance du langage par rapport à l'affect fait parfois peur, mais en explorant la valeur hypothétique des mots, Beckett ne fait que souligner la pertinence de la question essentielle de comment c'est. 